\title{
A NEW PUFF MODEL APPLYING AN EXACT SOLUTION OF DIFFUSION EQUATION
}

\author{
Everson Jonatha Gomes da Silva ${ }^{1}$, Tiziano Tirabassi², Daniela Buske ${ }^{3}$, Marco Túllio Menna \\ Barreto de Vilhena ${ }^{1}$ \\ ${ }^{1}$ UFRGS - PROMEC, Porto Alegre, Brasil \\ ${ }^{2}$ Institute ISAC of CNR, Bologna, Italy, t.tirabassi@isac.cnr.it \\ ${ }^{3}$ UFPel - IFM/DME, Pelotas, Brasil
}

\begin{abstract}
A new puff model is presented where the 3-dimensional puff diffusion is described by an analytical solution of the atmospheric diffusion equation.
\end{abstract}

\section{RESUMO}

Apresentamos um novo modelo tridimensional a puff onde a difusão é descrita por solução analítica da equação de difusão na atmosfera.

\section{INTRODUCTION}

The simplest approach in modelling unsteady conditions in both meteorology and emissions, is to describe the point source emission into the Planetary Boundary layer (PBL) by a series of instantaneous puffs, each one generated every time step and containing all the pollutant mass emitted during that time interval. Puff models are practical operative approach that simulate the pollutant dispersion in non-homogeneous and non-stationary atmospheric scenarios due to a source emission and they can operate both on short distance and mesoscale scenarios. The source emission is discretized in such successive set of puffs that each puff moves toward the area of calculus, driven by the three-dimensional wind profile which varies with time. Each puff is advected according to the local time varying mean flow and grows with time, depending on the turbulent properties of the PBL [1]. Puff models are quite suitable to handle the pollutant dispersion simulation in atmosphere over complex terrain. Their practical and theoretical simplicity, besides its flexibility are the reasons for the great success of this kind of model for pollutant dispersion.

Usually, the dispersion into the puffs are expressed by Gaussian formula, while in the present paper it is described by exacts solutions of the dispersion equation in the atmosphere obtained with the GILTT approach [2].

An example of application of the new model is presented.

\section{THE PUFF MODEL} follows:

Each puff has his trajectory described by its centre $\left(x_{0}, y_{0}, z_{0}\right)$ which is defined as

$x_{0}=\bar{u} \Delta t ; \quad y_{0}=\bar{v} \Delta t ; \quad z_{0}=\bar{w} \Delta t$

Where $\mathrm{z}$ is the vertical and $\mathrm{x}, \mathrm{y}$ the horizontal coordinates, $(\mathrm{u}, \mathrm{v}, \mathrm{w})$ is the wind velocity and $\Delta \mathrm{t}$ is the time interval.

Consequently the total concentration of a pollutant in a given point in space is given by the sum of all puffs issued, namely: 


$$
C_{T}(x, y, z, t)=\sum_{\text {puff }=1}^{\text {total of puffs }} \Delta M_{\text {puff }}\left\{\int_{t=0}^{\infty} c_{\text {puff }}(x, y, z, t) H\left(t-t_{0}\right) d t\right\}
$$

where $\mathrm{H}$ is the Heaviside function and $\Delta \mathrm{M}$ is the emission mass of every puff.

$c_{\text {puff }}(x, y, z, t)=c_{1}(x, t) c_{2}(y, t) c_{3}(z, t)$

As previously mentioned the three functions $c_{1}, c_{2}$ and $c_{3}$ are one-dimensional solution of the diffusion equation obtained by the GILTT approach [2].

Here, we assume that the solution is symmetric in the $\mathrm{x}$ and $\mathrm{y}$ directions. We must notice that $c_{i}$ denotes the mean concentration of a passive contaminant $\left(\mathrm{g} / \mathrm{m}^{3}\right), K_{x}, K_{y}$ and $K_{z}$ are the eddy diffusivities $\left(\mathrm{m}^{2} / \mathrm{s}\right)$ in the $x, y$ and $z$ directions, respectively, $\mathrm{Q}$ is the emission rate $(\mathrm{g} / \mathrm{s}), \mathrm{h}$ the height of the atmospheric boundary layer $(\mathrm{m}), \mathrm{H}_{\mathrm{s}}$ the height of the source $(\mathrm{m})$ and $\delta$ represents the Dirac delta function.

\section{DISCUSSION AND CONCLUSIONS}

Bearing in mind that the majority of puff models are based upon the Gaussian formulation for pollutant diffusion inside the puffs where the basic assumption is that puffs are dispersed by an homogeneous turbulence. Moreover, a distorting effect of the variation with height of the mean wind and eddy diffusivity, is often observed in the development of puffs. In fact, wind shear creates a concentration variance in the wind direction, while vertical diffusion destroys this concentration variance and tries to reestablish a nonskewed distribution.

In this work we have improved the puff model, in the sense that the pollutant dispersion is now described by the GILTT solution, based on a general technique for solving the diffusion equation, which is a proper solution either for homogeneous and nonhomogeneous scenarios in atmosphere and taking onto account eventually non-symmetries of puffs.. The solution simplicity comes from the fact that the three-dimensional solution is written as a product of one-dimensional ones.

We have applied the model using the Copenhagen experimental data set[3]. Generally, the distributed data set contains hourly mean values of concentrations and meteorological data. However, in this model validation, we used meteorology data with a greater time resolution kindly made available by Gryning and described in [4]. In particular, we used 10 minutes averaged values for meteorological data.

The model evaluation has to be considered preliminary because the data set considers neutral-convective ABL only.

Figure 1 shows the observed and predicted scatter diagram of predicted and measured data and the dotted line indicate the data that are in a factor two (that is, the values of predicted data are between half and twice as experimental ones). This figure, with the $67 \%$ of the data in a factor two, shows that a reasonable agreement is obtained between experimental data and the puff model.

Preliminary results against experimental data confirm the applicability of the approach proposed and are promising for future work. 


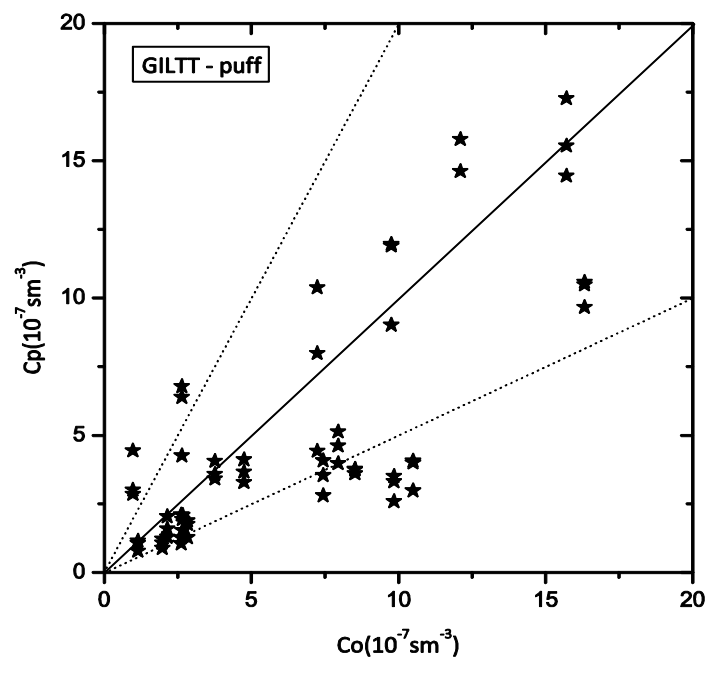

Figure 1.Observed and predicted scatter diagram of ground-level maximum crosswind concentrations using the GILTT-puff approach. Dotted lines indicate a factor of two

\section{REFERENCES}

[1] TIRABASSI T. "Mathematical Air Pollution Models: Eularian Models". In Air Pollution and Turbulenca - Modeling andApplications, edited by D. M. Moreira and M. Vilhena, CRC Press, New York (ISBN: 978-1-4398-1144-3), 324 p., 2010.

[2] Moreira D.M., Vilhena M.T., Buske D., Tirabassi T. The state-of-art of the GILTT method to simulate pollutant dispersion in the atmosphere. Atmos. Research 92, 1-17; 2009.

[3] Gryning, S.E. and Lyck, E. Atmospheric dispersion from elevated source in an urban area: comparison between tracer experiments and model calculations, J. Appl. Meteor. 23, 651-654; 1984.

[4] Tirabassi, T., Rizza, U. Boundary layer parameterization for a non-Gaussian puff model. J. Appl. Meteor. 36, 1031-1037, 1997. 